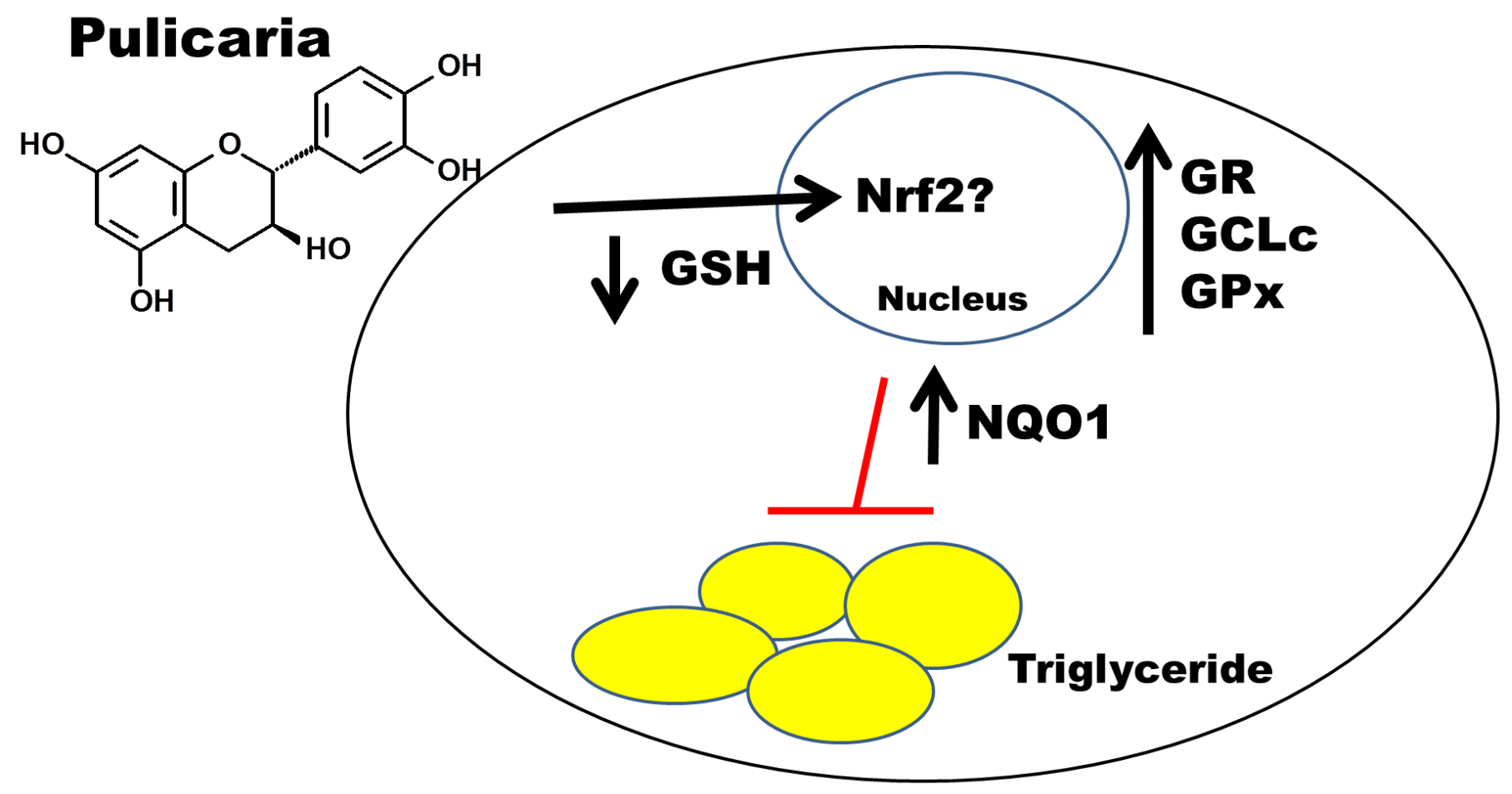

NQ01 (NADPH:Quinone OxidoReductase)1 GR (Glutathione Reductase)

GCLc (Glutamate Cysteine Ligase Catalytic Unit) GPx (Glutathione Peroxidase) 


\section{Pulicaria jaubertii E. Gamal-Eldin reduces triacylglyceride content and modifies cellular antioxidant pathways in 3T3-L1 adipocytes}

Ghanya Al-Naqeb ${ }^{1, \#}$, Jana Rousová ${ }^{2}$, Alena Kubátová2 ${ }^{2}$, Matthew J. Picklo, Sr. ${ }^{1,2^{*}}$

1 USDA-ARS Grand Forks Human Nutrition Research Center, Grand Forks, ND 58203 , USA ${ }^{2}$ Department of Chemistry, University of North Dakota, Grand Forks, ND, 58202, USA

*Corresponding author: USDA-ARS Grand Forks Human Nutrition Research Center, 2420 2nd Ave North, Grand Forks, ND 58203, United States.

Phone: 01-701-795-8380

Fax: 01-701-795-8240.

E-mail address: matthew.picklo@ars.usda.gov

\# Permanent address: Department of Food Sciences and Technology, Faculty of Agriculture, University of Sana'a, Yemen.

Funding was provided through USDA-ARS Project 5450-51000-048-00D. Dr. Al-Naqeb was the recipient of a Fulbright Scholarship that provided funding for this research. 


\section{Abstract}

Levels of obesity in Middle Eastern countries are increasing. Phytochemicals have antiobesogenic properties as evidenced by prevention of adipocyte differentiation and blocking triacylglyceride (TG) accumulation. In Yemen, Pulicaria jaubertii E.Gamal-Eldin (PJ) is a food additive and a traditional medicine. We tested the hypothesis that phytochemicals present in PJ inhibit adipocytic responses during differentiation of 3T3L1 preadipocytes to adipocytes. Methanolic extracts of PJ did not block expression of fatty acid binding protein 4 (FABP4) a marker of differentiation but did inhibit TG accumulation. Treatment of 3T3-L1 preadipocytes increased NADPH:quinone oxidoreductase 1 (NQO1), a suppressor of TG accumulation. Further fractionation of the methanolic PJ extract with hexane and dichloromethane (DCM) demonstrated that bioactivity towards TG reduction and elevated expression of NQO1 and other antioxidant genes (glutamate cysteine ligase catalytic unit, glutathione disulfide reductase, glutathione peroxidase (GPx) 4 resided in the DCM fraction. Activity towards depleting GSH and elevating the expression of catalase and GPx3 were found in the DCM and hexane fractions. Analysis by gas chromatography and liquid chromatography coupled with mass spectrometry demonstrated the presence of catechin-like moieties in the DCM and methanolic fractions and suggest that these components were partially responsible for the bioactivity of these fractions. In summary, our data indicate that fractions derived PJ exhibit anti-adipogenic properties in part through the presence of catechin-like compounds.

Keywords: 3T3-L1; Pulicaria jaubertii; NQO1; Glutathione; Catechin, Carvotanacetone, Adipocyte 


\section{Abbreviations}

3-(4,5-dimethylthiazol-2-yl)-2,5-diphenyltetrazolium bromide (MTT)

Dichloromethane (DCM)

Dimethylsulfoxide (DMSO)

Electrospray ionization with high-resolution time of flight mass spectrometry (ESI-TOFMS)

Fatty acid binding protein (FABP)

Gas chromatography-mass spectrometry (GC-MS)

Glutamate cysteine ligase catalytic unit (GCLc)

Glutathione (GSH)

Glutathione disulfide (GSSG)

Glutathione peroxidase (GPx)

Glutathione reductase (GR)

Liquid chromatography-mass spectrometry (LC-MS)

NADPH:quinone oxidoreductase 1 (NQO1)

N,O-bis(trimethylsilyl)trifluoroacetamide (BSTFA)

Pulicaria jaubertii Gamal-Eldin (PJ)

Pulicaria jaubertii methanol extract $\left(\mathrm{PJ}_{\mathrm{M}}\right)$

Pulicaria jaubertii hexane fraction $\left(\mathrm{PJ}_{\mathrm{H}}\right)$

Pulicaria jaubertii dichloromethane fraction $\left(P J_{D}\right)$

Pulicaria jaubertii aqueous fraction $\left(\mathrm{PJ}_{\mathrm{A}}\right)$

Superoxide dismutase (SOD)

Triacylglyceride (TG)

Trimethylsilyl (TMS) 


\section{Introduction}

In the Middle East, levels of obesity are growing along with the predicted sequelae like insulin resistance $[1,2]$. These events have resulted in an increasing attention towards the identification of the anti-obesigenic properties of traditional Middle Eastern herbal medicines. Species of the genus Pulicaria are widespread throughout the Mediterranean, Middle East, and Asia, and their extracts are used as traditional medicines [3]. Pulicaria jaubertii Gamal-Eldin (PJ) is a Yemeni flavorant and is used in traditional medicines [4, 5]. The extracted oils of PJ have cytotoxic effects towards MCF-7 cancer cells and moderate antimicrobial activities [6]. The extent to which PJ contains phytochemicals with anti-obesogenic properties has not been explored.

Multiple reports indicate that phytochemicals modify adipogenic responses [7-9]. For example, curcumin, isolated from turmeric, inhibits adipocyte differentiation [10]. Several phytochemicals have been studied for their anti-adipogenic activity in vivo and in vitro using the murine 3T3-L1 adipocyte model. Our previous work demonstrates that elevated expression of NADPH:quinone oxidoreductase 1 (NQO1) by sulforaphane, a isothiocyanate derived from brassicates, inhibits triacylglyceride (TG) accumulation in 3T3-L1 adipocytes [11].

Mechanistic evidence indicates that adipocyte differentiation and TG accumulation are controlled by the cellular redox status and the expression of antioxidant enzymes. NQO1 is part of the antioxidant defense system and is extensively studied in relation to chemoprotection and cancer susceptibility $[12,13]$. Multiple lines of evidence indicate 
that NQO1 plays a mechanistic role in adipocyte function and energy homeostasis. The NQO1 gene is highly expressed in human adipose $[14,15]$. NQO1 deficiency modifies abdominal adipose tissues mass in mice [16]. Moreover, the expression of NQO1 is down-regulated during the differentiation of 3T3-L1 preadipocytes into adipocytes and enhancing NQO1 inhibits differentiation [11].

Glutathione $(\mathrm{GSH})$ is one of the most important anti-oxidants in the cell and its roles in obesity and energy metabolism have recently been reviewed [17]. GSH has many functions in regulating the development of obesity. In vitro mechanistic studies using 3T3-L1 preadipocytes demonstrate that GSH depletion with buthionine sulfoximine induces adipogenesis and TG deposition, an effect reversed by GSH ethyl ester [18]. Similarly, $\mathrm{N}$-acetyl-cysteine, a GSH precursor, inhibits adipogenesis [19]. Conversely, depletion of the glutathione by BSO in mice prevents diet-induced obesity, increases energy expenditure and enhances insulin sensitivity [20]. Data suggest that GSH depletion in vivo may increase energy expenditure and decrease adiposity via Nrf2ARE-based mechanisms as reviewed recently [17, 21].

With these data in mind, we tested the hypothesis that phytochemicals present in PJ inhibit adipocytic responses during differentiation of 3 T3-L1 preadipocytes to adipocytes. Our results showed that methanolic extracts of PJ blocked accumulation of TG but not differentiation. These effects occurred in the presence of GSH depletion and increases in several antioxidant defense enzymes including NQO1, glutathione peroxidase 3 (GPx3), GPx4, GR, glutamate-cysteine ligase (GCL) and catalase. 
Analysis of extract fractions by gas chromatography-mass spectrometry (GC-MS) and liquid chromatography-mass spectrometry (LC-MS) identified multiple compounds with potential biological activity.

\section{Materials and methods}

2.1 Plant collection. Pulicaria jaubertii E.Gamal-Eldin was collected from Alkaa Farm, located near Sana`a, Yemen, in September 2013. The plant was identified and authenticated by Dr. Abdul Wali Al Khulaidi, a plant taxonomist at the Department of Botany, Faculty of Agriculture, Sana`a University, Yemen. Leaves and flowers were separated from the stems. Leaves and flowers were naturally dried, packed in plastic bags, and stored at $4^{\circ} \mathrm{C}$ with protection from light. The dried material was sent to the USDA-Grand forks Human Nutrition Research Center, in Grand Forks, North Dakota, USA, in December 2013.

2.2 Chemicals. Methanol (LC-MS Optima grade), and dichloromethane (DCM, GC quality) were purchased from Fisher Scientific. n-Hexane (LC-MS grade) was obtained from JT Baker, USA. N,O-bis(trimethylsilyl)trifluoroacetamide (BSTFA, 99\%) with 1\% trimethylchlorosilane (TMCS) was purchased from Sigma-Aldrich (St. Louis, MO, USA) . and was used to form trimethylsilyl (TMS) derivatives.

2.3 Preparation of the PJ extract and its fractionation. Dried PJ was finely ground using an electric grinder (Ika Labortechnik M 20 Brand). The resulting green powder was extracted with methanol at 1:5 powder:methanol $(\mathrm{w} / \mathrm{v})$ ratio for $48 \mathrm{~h}$ with stirring under dark conditions. The resulting extract was filtered, and the resulting filtrate concentrated 
by rotary evaporation at $40^{\circ} \mathrm{C}$. The final, dark green viscous extract was transferred into glass amber bottles and stored at $4{ }^{\circ} \mathrm{C}$ for subsequent analyses. A $5 \%$ yield of final methanolic $\mathrm{PJ}$ extract, denoted $P \mathrm{~J}_{\mathrm{M}}$, relative to starting powder was obtained.

Twelve (12) $\mathrm{g}$ of $P J_{M}$ was mixed with $n$-hexane at a $1: 5 \mathrm{w} / \mathrm{v}$ ratio and $100 \mathrm{~mL}$ of methanol: water $(80: 20 \mathrm{v} / \mathrm{v})$ was added. The mixture was shaken in a separatory funnel and the hexane layer was removed. This step was repeated once more. Dichloromethane (DCM) was added into the remaining aqueous residue and the process repeated. The resulting DCM and hexane fractions were concentrated to dryness using a rotary evaporator. Both fractions were highly viscous and dark green in color. The aqueous phase was subjected to freeze drying yielding a viscous, dark amber residue. The yields obtained for each PJ fraction with respect to the initial methanolic extract were: $n$-hexane (denoted $\left.\mathrm{PJ}_{\mathrm{H}}\right) 5 \mathrm{~g}(41 \%)$, DCM (denoted PJD) $2 \mathrm{~g}$ (16\%) and residual methanol:aqueous fraction (denoted $\left.P J_{A}\right) 1 \mathrm{~g}(8 \%)$.

2.4 Chemical characterization of PJ fractions. For GC-MS analysis, the methanolic extract, $P J_{M}$, and each fraction $\left(P J_{H}, P J_{D}, P J_{A}\right)$ were analyzed in amounts proportional to the weight of fraction obtained from the extract (i.e., $5 \mathrm{mg}$ of $P J_{H}, 1.8 \mathrm{mg}$ of $P J_{D}$ ). Samples were dissolved in $1 \mathrm{~mL}$ DCM with and without TMS derivatization with BSTFA. Prior to GC-MS analyses, $25 \mu \mathrm{L}$ of internal standard (o-terphenyl, $1.4 \mathrm{mg} / \mathrm{mL}$ ) was added to control for volume changes in each sample. The tentative identification of observed compounds was based on comparison with the NIST 05 Mass Spectra library. 
For TMS derivatization, appropriate amounts of the methanol extract and its fractions were weighed, dried, and reconstituted in100 $\mu \mathrm{L}$ of BSTFA and $50 \mu \mathrm{L}$ pyridine. The solution was heated at $70^{\circ} \mathrm{C}$ for $18 \mathrm{~h}$. After derivatization $25 \mu \mathrm{L}$ of internal standard (oterphenyl) was added together with $0.85 \mathrm{~mL}$ of DCM.

GC-MS analyses were performed using 6890N GC with 5975C MS (Agilent Technologies, Santa Clara, CA, USA) equipped with Gerstel MPS2 autosampler (Gerstel, Baltimore, MD, USA). Injections (1 $\mu \mathrm{L})$ were performed in a splitless mode for $0.40 \mathrm{~min}$ at $250^{\circ} \mathrm{C}$. Separation was performed using a DB-5MS capillary column (30 $\mathrm{m}$ ), with $0.25 \mathrm{~mm}$ internal diameter (I.D.) and $0.25 \mu \mathrm{L}$ film thickness (J\&W Scientific, Folsom, CA, USA). A constant carrier gas (helium) flow rate of $1 \mathrm{~mL} / \mathrm{min}$ was maintained during the analysis. The temperature program started at $35^{\circ} \mathrm{C}$ held for 5 min, followed by a gradient of $10{ }^{\circ} \mathrm{C} / \mathrm{min}$ to $300^{\circ} \mathrm{C}$ and held for $1 \mathrm{~min}$. The MS data in total ion chromatogram (TIC) mode were acquired in the mass range of $m / z$ of $35-1000$ at a scan rate $2.66 \mathrm{scan} / \mathrm{s}$ using the $\mathrm{El}$ of $70 \mathrm{eV}$. The solvent delay was based elution of the derivatization agent, their by-products, and solvent retention times, measured by observing the vacuum change. Namely, for BSTFA with pyridine, the MS was off for the first $2.5 \mathrm{~min}, 2.90-3.60 \mathrm{~min}, 4.40-7.00 \mathrm{~min}, 8.00-8.70 \mathrm{~min}$; for samples without derivatization, solvent delay was $2.5 \mathrm{~min}$.

For LC-MS analyses, a stock solution of each sample $\left(P J_{M}, P J_{H}, P J_{D}, P J_{A}\right)$ was prepared in methanol in concentration of $2-3 \mathrm{mg} / \mathrm{mL}$. Stocks were further diluted to final concentration of $10-16 \mathrm{ppm}$ in acetonitrile and water $(50: 50 \mathrm{v} / \mathrm{v})$ with ammonium 
acetate (final concentration of $5 \mathrm{mM}$ ). LC-MS analyses were carried out using electrospray ionization with high-resolution time of flight mass spectrometry (ESI-TOFMS, G1969A, Agilent, Santa Clara, CA, USA) coupled to an Agilent LC 1100 high performance liquid chromatograph. The column was a $\mathrm{C}_{18}$ Zorbax Eclipse plus $(2.1 \mathrm{x}$ $150 \mathrm{~mm}$ with $3.5 \mu \mathrm{m}$ particle size) with a $1.2 \mathrm{~cm}$ guard column (Agilent). For the chromatographic separations, the mobile phase solutions consisted of $1.0 \mathrm{mM}$ ammonium acetate in water:acetonitrile $(95: 5, \mathrm{v} / \mathrm{v})$ as solvent $A$ and $1.0 \mathrm{mM}$ ammonium acetate in acetonitrile as solvent $\mathrm{B}$. The flow rate was $0.2 \mathrm{~mL} / \mathrm{min}$, and the injection volume was $50 \mu \mathrm{L}$ with an injector needle wash program to avoid cross-contamination. The gradient elution program started isocratically at $0 \% \mathrm{~B}$ for $2 \mathrm{~min}$, followed by a linear gradient to $80 \%$ B from 2 min to 30 min with maintenance of $80 \%$ B for 5 min. Following a sharp gradient to $100 \% \mathrm{~A}$ from 35 to $36 \mathrm{~min}$, the column was re-equilibrated with $100 \%$ solvent A from 36 to $56 \mathrm{~min}$. The column temperature was maintained at $30^{\circ} \mathrm{C}$. ESI was performed in negative mode with an electrospray voltage of $4500 \mathrm{~V}$, a fragmentor voltage of $175 \mathrm{~V}$, a nebulization pressure of $25 \mathrm{psig}$, a drying gas flow rate of $12 \mathrm{~L} / \mathrm{min}$, and a drying gas temperature of $350^{\circ} \mathrm{C}$.

\subsection{Cell culture.}

Mouse 3T3-L1 preadipocytic fibroblasts were obtained from American Type Culture Collection (ATCC; Manassas, VA). Cells were cultured as previously described [11]. For cell viability determination, the 3T3-L1 preadipocytes were seeded at a density of $6 \mathrm{X}$ $10^{3}$ cells/well in 24 well plates. The cells were then treated with increasing doses of extracts for $24 \mathrm{~h}$. Dried extracts and fractions were dissolved in dimethylsulfoxide 
(DMSO). The DMSO percentage was $<0.1 \%$ at final concentration. Cell viability was determined using the 3-(4,5-dimethylthiazol-2-yl)-2,5-diphenyltetrazolium bromide (MTT) activity assay as described previously [22].

Differentiation of 3T3-L1 pre-adipocytes to adipocytes occurred through a seven-day treatment regimen using insulin, dexamethasone, and isobutylmethylxanthine as previously described [11]. For experiments testing the inhibition of adipocyte differentiation, confluent 3T3-L1 pre-adipocytes were treated with selected doses of the extracts at days $0,2,5$, and 7 that corresponded to changes in the cell culture media. PJ fractions were diluted in DMSO with final concentrations of DMSO $<0.1 \%$.

2.5.1 Oil red O staining. Lipid accumulation in differentiated adipocytes was assessed by Oil Red O staining [23]. Cells (plated in 6 well plates) were washed with phosphatebuffered saline and fixed with $10 \%$ formalin for $30 \mathrm{~min}$. After two washes with distilled water, cells were washed with $60 \%$ isopropanol and allowed to completely dry at room temperature. Cells were treated with an Oil Red $O$ working solution for 10 min with rocking. Following incubation of the cells, the Oil Red O solution was removed, and the cells immediately washed four times with distilled water. After the final wash, distilled water was added to each well prior to microscopy. The Oil Red O working solution was made by (1) dissolving $0.35 \mathrm{~g}$ of Oil Red O (Sigma-Aldrich, St. Louis, MO) in $100 \mathrm{~mL}$ of isopropanol with stirring overnight followed by filtration to make an initial stock solution and (2) diluting $6 \mathrm{~mL}$ of this stock solution with $4 \mathrm{~mL}$ of distilled water. 
2.5.2 Triacylglyceride (TG) analysis. Determination of cellular TG content was performed as described previously [11]. Cells were washed with PBS and lipids were extracted with hexane: isopropanol at 3:2 v/v. Extracted lipids were dissolved in methanol and the TG content was determined on the Roche COBAS Integra 400+ (Roche Diagnostics, Indianapolis, IN). The resulting protein cell pellet was dried at 65 ${ }^{\circ} \mathrm{C}$ for $24 \mathrm{~h}$, re-suspended in $1 \mathrm{M}$ sodium hydroxide and analyzed for protein content. Protein was quantified using the Bio-Rad Protein Dye Kit (Bio-Rad, Hercules, CA) using bovine serum albumin as a standard. TG content was normalized to the cellular protein content. Results are expressed as total mg TG/mg protein.

2.5.3 Leptin determination. Leptin concentration in cell culture media was determined by a mouse-specific enzyme-linked immunosorbent assay (Abcam Cambridge, MA). Briefly, this assay employed an antibody specific for mouse leptin coated on a 96- well plate. Recombinant mouse leptin was used as a standard. All samples were measured in duplicate. Leptin content was normalized to the cellular protein content of the harvested cells.

2.5.4 Gene expression analysis. For determination of gene expression, RNA was isolated using the QIAcube system (Qiagen, Valencia, CA), consisting of a QIA shredder spin column, an RNeasy MiniKit, and on-column DNAse I treatment, following the manufacturer's protocols. The quantity and quality of RNA were determined on a NanoDrop 8000 spectrophotometer (Thermo Fisher Scientific, Wilmington, DE). RNA $(1 \mu \mathrm{g})$ was converted to cDNA using the GoScript reverse transcription system kit 
(Promega, Madison, WI). The sequences of the primers used in the study are presenting in Table 1. Real time PCR reactions contained the following: $1 \mathrm{X}$ SYBR Green Master Mix (Applied Biosystem, Inc.,Carlsbad, CA), 250 nM of forward and reverse primers $1 \mu \mathrm{g}$ CDNA. The final volume of the reactions was $50 \mu \mathrm{L}$ and dissociation curves confirmed a single amplification species. Real Time PCR was performed on a 7500 Fast Real Time PCR System (Applied Biosystem, Inc.,Carlsbad, CA). Real-time qPCR primers were designed and purchased from IDT (Coralville, IA). Expression of candidate genes was normalized to $\beta$-actin expression levels.

2.5.5 Immunoblotting of NQO1 and fatty acid binding protein (FABP) 4. Cells were washed with PBS and homogenized with cold homogenizing buffer (20 mM sodium phosphate, $1 \mathrm{mM}$ diethylene triamine pentaacetic acid (DTPA), $0.05 \mathrm{mM}$ butylated hydroxytoluene $(\mathrm{BHT})$, and $0.5 \%$ Triton $\mathrm{X}-100, \mathrm{pH} 7.4$, and $1 \%$ Protease Inhibitor Cocktail (Sigma Aldrich, St. Louis, MO)) and sonicated at 3 bursts for $10 \mathrm{~s}$ each at $50 \%$ amplitude with a sonic dismembrator (Fisher Scientific, Waltham, MA). Total protein was determined using the Bio-Rad protein dye kit (Bio-Rad, Hercules, and CA) using bovine serum albumin as the standard. Immunoblot analysis for NQO1 and FABP4 was performed according to Vomhof-DeKrey and Picklo [11].

2.5.6 GSH and glutathione disulfide (GSSG) analyses. Experiments were performed in $25 \mathrm{~cm}^{2}$ flasks. After completion of the treatment period, the cell medium was aspirated and the cells were washed with ice-cold PBS. Cells were scraped into $250 \mu \mathrm{L}$ of $5 \%$ sulfosalicylic acid (Sigma-Aldrich, St. Louis, MO; dissolved in water) and sonicated. The 
resulting homogenate was centrifuged at $14000 \times \mathrm{g}$ for $10 \mathrm{~min}$ at $4{ }^{\circ} \mathrm{C}$. The supernatant was removed and assayed for GSH and GSSG content and the pellet was assayed for total protein. GSH and GSSG content were determined using a modification of the method reported by Dringen and colleagues [22, 24].

2.6 Statistical analysis. Statistical comparisons were made using Prism 5.0 software (GraphPad Software, San Diego California USA, www.graphpad.com). Data were analyzed using one-way ANOVA and statistical significance was taken as $p \leq 0.05$. All data are expressed as the mean \pm the standard deviation (SD) unless otherwise stated.

\section{Results}

\subsection{Inhibition of TG accumulation}

In an initial set of experiments, the effect of $P J_{M}$ upon cell viability, as determined by the MTT assay, was assessed in order to select $P J_{M}$ concentrations for subsequent analyses. Exposure of 3T3-L1 pre-adipocytes to $P J_{M}$ for 24 hrs resulted in a dosedependent decrease in MTT activity (Figure 1). We arbitrarily set the highest concentration of $P J_{M}(55 \mu \mathrm{g} / \mathrm{mL})$ used for subsequent experiments to be the concentration which retained $80 \%$ of control MTT activity.

The ability of $P J_{M}$ to inhibit lipid accumulation was evaluated histochemically through Oil Red $\mathrm{O}$ staining and through analysis of cellular TG content. Confluent monolayers of 3T3-L1 pre-adipocytes were treated with increasing concentrations of freshly-diluted 
$P J_{M}$ on days $0,2,5$, and 7 , days that corresponded to changes in the cell culture media. The resulting differentiated adipocyte cultures were fixed or harvested on day 8 . At day 8, untreated differentiated cells had accumulated lipid droplets as demonstrated by Oil Red $\mathrm{O}$ staining (Figure $2 \mathrm{~A}$ ), an effect inhibited by $P \mathrm{~J}_{\mathrm{M}}$ treatment. In agreement with the staining results, $P J_{M}(10 \mu \mathrm{g} / \mathrm{mL})$ lowered TG content by approximately $30 \%$ in the differentiated adipocyte cultures (Figure 2B).

Increased expression of FABP4 is recognized as a marker of adipocytic differentiation [25]. We tested whether $P J_{M}$ treatment blocked differentiation of preadipocytes to adipocytes vs blocking TG accumulation. As expected FABP4 content increases with differentiation of preadipocytes to adipocytes (Figure 2C). In contrast to its effects on $T G, P J_{M}$ did not block expression of FABP4, indicating that $P J_{M}$ does not block adipocyte differentiation.

That adipocyte differentiation is not blocked by $P J_{M}$ is further supported by analysis of leptin secretion. Following differentiation the leptin concentration in the cellular medium more than doubled for differentiated 3T3-L1 adipocytes undifferentiated 3T3-L1 preadipocytes (Figure 2D). While there was a reduction in leptin secretion as a result of $P J_{M}$ exposure, leptin levels were nearly $60 \%$ greater in adipocytes treated with $P J_{M}$ vs pre-adipocytes.

\subsection{Changes in redox markers}


Previous data shows that suppression of TG content in 3T3 adipocytes by the phytochemical sulforaphane occurs in part through elevating the expression of the antioxidant enzyme NQO1 [11]. In this experiment, we tested the extent to which $P J_{M}$ increases the expression of NQO1 (Figure 3). NQO1 gene expression was elevated 5fold in in preadipocytes treated for $48 \mathrm{~h}$ with $\mathrm{PJ}_{\mathrm{M}}$ (Figure $3 \mathrm{~A}$ ). Moreover, in preadipocytes treated with $\mathrm{PJ}_{\mathrm{M}}$ during the seven-day differentiation protocol, NQO1 protein content was elevated $>10$-fold by $\mathrm{PJ}_{\mathrm{M}}$ treatment vs untreated adipocytes or preadipocytes (Figure 3B).

Elevated expression of NQO1 can occur through stimulation of the Nrf2-ARE pathway that can be activated by depletion of $\mathrm{GSH}[13,26]$. Following a six-hour treatment with $\mathrm{PJ}_{\mathrm{M}}, \mathrm{GSH}$ content in preadipocytes was depleted in a dose-dependent manner (Figure 4A). Conversely, the GSSG level (Figure 4B) was increased by $P J_{M}$ when compared to control cells. When the GSH: GSSG ratio was calculated, we observed decrease in this ratio starting at $10 \mu \mathrm{g} / \mathrm{mL}$ of $P J_{M}$ (Figure $4 \mathrm{C}$ ) compared to control cells.

\subsection{Determination of bioactive fractions}

In order to discern the bioactive components responsible for changes noted above, $P J_{M}$ was fractioned into $n$-hexane $\left(P J_{H}\right)$, dichloromethane $\left(P J_{D}\right)$ and aqueous $\left(P J_{A}\right)$ fractions. The bioactivity of the resulting fractions was determined using a concentration of fraction comparable to that present in the starting $P J_{M}$. Thus, $P J_{M}$ at $55 \mu \mathrm{g} / \mathrm{mL}, P J_{H}$ at $25 \mu \mathrm{g} / \mathrm{mL}$ and $P J_{D}$ at $10 \mu \mathrm{g} / \mathrm{mL}$ were used in the subsequent assays. $P J_{A}$ was used 10 $\mu \mathrm{g} / \mathrm{mL}$, a concentration twice that found in $P J_{M}$. 
We examined the extent to which inhibition of TG accumulation was present in the resulting PJ fractions by treatment of preadipocytes during the seven-day differentiation protocol used above. Our results (Table 2) show that the $P J_{D}$ but not the $P J_{H}$ or $P J_{A}$ fractions contained significant activity towards inhibiting TG accumulation. On the other hand, $P J_{D}$ and $P J_{H}$ fractions both contained compounds that resulted in depletion of GSH.

We further examined the extent to which the $P J_{H}, P J_{D}$, and $P J_{A}$ fractions modified the expression of NQO1 and other genes that contribute to changes in cellular redox status and anti-oxidant defense responses (Table 2). In these experiments, 3T3-L1 preadipocytes were treated with the fraction and harvested $48 \mathrm{hrs}$ later, similar to experiments described above. $\mathrm{PJ}_{\mathrm{M}}$ elevated the expression of $\mathrm{NQO1}$, glutathione disulfide reductase $(G R)$, glutamate cysteine ligase catalytic subunit $\left(G C L_{C}\right)$, glutathione peroxidase 3 (GPx3), GPx4, and catalase. This pattern was replicated by the dichloromethane fraction, $P J_{D}$. The $P J_{H}$ fraction, on other hand, only induced expression of GPx3 and catalase. The final, aqueous fraction $\mathrm{PJ}_{\mathrm{A}}$ did not affect the gene expression endpoints examined. Expression of GPx1, SOD1 and SOD2 was not modified by any fraction.

\subsection{Chemical characterization of PJ fractions}

The $P J_{M}, P J_{H}, P J_{D}$, and $P J_{A}$ fractions were analyzed by GC-MS and LC-TOF-MS in order to characterize the bioactive compounds present. GC-MS analyses were 
performed directly on the fractions or following TMS derivatization. The formation of TMS derivatives allowed for analysis of hydroxy- and carboxy-containing compounds with limited volatility. Direct GC-MS analysis (without TMS derivatization) (Figure 5) of PJ fractions showed that most fractions contained carvotanacetone, with the highest abundance in $\mathrm{PJ}_{\mathrm{H}}$. Other compounds were observed in limited abundance and are further detailed in supplementary data (Table S1). These results are in agreement with prior reports of the chemical composition of other Pulicaria species $[4,6,27,28]$.

In order to further characterize less volatile, polar components by GC-MS, TMS derivatization was performed (Figure 6). Based upon the match with the NIST mass spectra library, catechin-like compounds were found in high-abundance in the $P J_{M}$ extract and the $P J_{D}$ fractions (Figures $6 A, C$ ). These results parallel the bioactivity of these two fractions. Several other compounds, including carvotanacetone, fatty acids and sugars were observed in the fractions analyzed and are further detailed in supplementary data (Table S2).

Further analysis of these catechin-like compounds was performed (Figure 7). Focusing in on the relevant section of the chromatogram derived from GC-EI/MS of TMSderivatized $P J_{D}$ fraction, six catechin-like compounds were observed (Figure $7 A$ ). Three of these compounds, peaks D2, D3, D4 (Figure 7B-D), had a characteristic common ion $368 \mathrm{~m} / \mathrm{z}$ that matched the El mass spectra of catechin and epi-catechin analytical standards (Figure 7E) [29]. However, the retention time of these catechin-like species 
did not match the retention time of the catechin and epicatechin standards (Figure 8). The low resolution of the EI-MS data prevented further identification of these compounds.

The $P J_{D}$ fraction was analyzed using LC with high resolution TOF-MS. Similar to GCMS, two peaks with ions of $289.07 \mathrm{~m} / \mathrm{z}$ (corresponding to $\left[\mathrm{M}^{-} \mathrm{H}^{-}\right]$ion for catechin) were observed (Figure 9A), and similar to GC, they eluted at later retention times. The low mass accuracy error of $14 \mathrm{ppm}$ supported the molecular structure of this ion as the same as that of catechin (Figure 9A). The mass spectra of these peaks (Figure 9B,C) showed the most abundant fragments as $317.05 \mathrm{~m} / \mathrm{z}$ and $359.19 \mathrm{~m} / \mathrm{z}$. The first peak $\left(L 1, t_{R} 19.15\right)$ with the ions of $289 \mathrm{~m} / z$ and $317 \mathrm{~m} / \mathrm{z}$ may be related to GC-MS peaks D2 and D4 (Figure 7), respectively. The $317 \mathrm{~m} / \mathrm{z}$ corresponds to catechin with a CO group, with 40 ppm error. The second peak (L2) had an abundant ion of $359 \mathrm{~m} / \mathrm{z}$, suggesting that the parent molecule possessed a mass of 360 with a proposed structure $\mathrm{C}_{20} \mathrm{H}_{24} \mathrm{O}_{6}$ with 111 ppm error.

\section{Discussion}

Obesity is a global health concern, including in the Middle East. Therapeutic strategies include nutritional, pharmacological, and surgical interventions. Phytochemicals from traditional medicinal herbs offer an alternative approach. Pharmacological characterization of Pulicaria jaubertii E.Gamal-Eldin (PJ) is lacking even though it has been used by the Yemeni people as a traditional medicine. In this study, we examined the anti-obesogenic properties of PJ. Our data demonstrate that organic extracts of PJ attenuated TG accumulation and increased the expression of multiple antioxidant 
genes. Chemical fractionation and MS-based characterization indicate the catechin-like compounds present in the DCM fraction of PJ are responsible in part for this activity.

$P J_{M}$ blocks accumulation of $T G$ but not expression of the adipocyte differentiation marker FABP4. FABP4 is a key factor for intracellular fatty acid transport and lipid metabolism [30]. These data indicate that decreases in TG accumulation were not the result of inhibiting differentiation of the pre-adipocytes into adipocytes per se but by inhibition of TG accumulation itself. Treatment of 3T3-L1 preadipocytes with epigallocatechin yields a similar response [31]. These data are in contrast to other phytochemicals like sulforaphane and curcumin that inhibit TG synthesis in addition to inhibiting expression of FABP4 [11, 23].

$\mathrm{PJ}_{\mathrm{M}}$ increased the expression of several genes NQO1, GR, GCL $L_{C}$, and $\mathrm{GPx} 4$ associated with antioxidant defense and activation of the Nrf2-ARE pathway [32]. Previous data from this laboratory demonstrate the isothiocyanate sulforaphane, a known activator of the Nrf2-ARE, inhibits TG accumulation in part through elevation of NQO1 activity [11]. Given these previous data, our findings suggest that activation of the Nrf2-ARE pathway contributed to the decrease in TG accumulation. While our experiments do not specifically test for activation of an Nrf2-ARE reporter or movement or accumulation of Nrf2 to the nucleus, the activation of a number of Nrf2-ARE inducible gene tested strongly support the activation of this pathway 
The role of GSH in adipogenesis and obesity development is complex [17]. Previous in vitro experiments demonstrate that GSH depletion increases adipogenesis and TG accumulation and that supplementation with GSH precursors can block adipogenesis $[18,19]$. Our data do not support these findings since $\mathrm{PJ}_{H}$ while reducing cellular $\mathrm{GSH}$ did not significantly modify (increase or decrease) TG accumulation. We recognize however that our experiments utilized a complex mixture of bioactive compounds with potential pro-adipogenic and anti-adipogenic properties. In this context, GSH depletion itself may not be sufficient to modulate TG accumulation.

Consistent with activity in $P J_{M}$, the $D C M$ fraction $P J_{D}$ contained catechin-like compounds to a similar degree as found in $P J_{M}$. The effect of catechin species in preventing the differentiation of adipocytes is well-documented [31, 33-35]. Catechins and related flavonoid phytochemicals have not been reported in previous analyses of PJ, likely as the result of using GC-MS without TMS derivatization. However, a number of flavonoids and sterols have been detected in other Pulicaria species [3].

GC-MS analysis of $P J_{M}$ and the $P J_{H}$ and $P J_{D}$ fractions resulted in the identification of carvotanacetone as being a major component. Carvotanacetone is an $\alpha, \beta$-unsaturated ketone. Given its structure, carvotanacetone has an electrophilic center and thus likely can form a Michael adduct with GSH. While carvotanacetone is not commercially available, treatment of 3T3-L1 cells with the structural analog carvone confirms that carvone-type compounds deplete cellular GSH (data not shown). 
In conclusion, this study provides in vitro evidence of anti-adipogenic properties of Pulicaria jaubertii E.Gamal-Eldin. Our results show that the methanol extract of PJ and its subsequent DCM fraction, likely through the presence of catechin-like compounds, possessed bioactivity towards inhibiting TG accumulation in adipocytes. Our data provide new knowledge regarding the abundance of compounds in this Yemeni herb and their ability to modify cellular anti-oxidant properties.

\section{Acknowledgments}

The authors are grateful to Mary Briske-Anderson, Kim Michelson, Gregoire Brian and Dale Christopherson for their technical assistance with this work. Funding was provided through USDA-ARS Projects 5450-51000-048-00D. The U.S. Department of Agriculture, Agricultural Research Service, Plains Area is an equal opportunity/affirmative action employers and all agency services are available without discrimination. Mention of trade names or commercial products in this article is solely for providing specific information and does not imply recommendation or endorsement by the U.S. Department of Agriculture. 


\section{References}

[1] M. Ng, T. Fleming, M. Robinson, B. Thomson, N. Graetz, C. Margono, E.C. Mullany, et al., Global, regional, and national prevalence of overweight and obesity in children and adults during 1980-2013: a systematic analysis for the Global Burden of Disease Study 2013, Lancet 384 (2014) 766-781.

[2] B.M. Popkin, M.M. Slining, New dynamics in global obesity facing low- and middle-income countries, Obes Rev 14 Suppl 2 (2013) 11-20.

[3] L.L. Liu, J.L. Yang, Y.P. Shi, Phytochemicals and biological activities of Pulicaria species, Chem Biodivers 7 (2010) 327-349.

[4] M.N. Algabr, A. S., A. Menad, R. Mekkiou, J.C. Chalchat, B. S., F. Benayache, Essential oil composition of Pulicaria jaubertii from Yemen, Int $\mathrm{J}$ Med Arom Plants 2 (2012) 688-690.

[5] A.S. Dubaie, A.A. Al-Khulaida, Studies on the Flora of Yemen On the flora of Tihama plain J Botan Taxon Geobot 104 (1993) 259-265.

[6] G.A. Fawzy, H.Y. Al Ati, A.A. El Gamal, Chemical composition and biological evaluation of essential oils of Pulicaria jaubertii, Pharmacogn Mag 9 (2013) 2832.

[7] M. Gonzalez-Castejon, A. Rodriguez-Casado, Dietary phytochemicals and their potential effects on obesity: a review, Pharmacol Res 64 (2011) 438-455. 
[8] J. Lin, M.A. Della-Fera, C.A. Baile, Green tea polyphenol epigallocatechin gallate inhibits adipogenesis and induces apoptosis in 3T3-L1 adipocytes, Obes Res 13 (2005) 982-990.

[9] N. Siriwardhana, N.S. Kalupahana, M. Cekanova, M. LeMieux, B. Greer, N. Moustaid-Moussa, Modulation of adipose tissue inflammation by bioactive food compounds, J Nutr Biochem 24 (2013) 613-623.

[10] A. Ejaz, D. Wu, P. Kwan, M. Meydani, Curcumin inhibits adipogenesis in 3T3-L1 adipocytes and angiogenesis and obesity in C57/BL mice, J Nutr 139 (2009) 919925.

[11] E.E. Vomhof-DeKrey, M.J. Picklo, NAD(P)H:quinone oxidoreductase 1 activity reduces hypertrophy in 3T3-L1 adipocytes, Free Radic Biol Med 53 (2012) 690700.

[12] A.K. Jaiswal, Regulation of genes encoding $\operatorname{NAD}(\mathrm{P}) \mathrm{H}$ :quinone oxidoreductases, Free Radic Biol Med 29 (2000) 254-262.

[13] D. Ross, J.K. Kepa, S.L. Winski, H.D. Beall, A. Anwar, D. Siegel, $\mathrm{NAD}(\mathrm{P}) \mathrm{H}$ :quinone oxidoreductase 1 (NQO1): chemoprotection, bioactivation, gene regulation and genetic polymorphisms, Chem Biol Interact 129 (2000) 7797.

[14] J. Palming, K. Sjoholm, M. Jernas, T.C. Lystig, A. Gummesson, S. Romeo, L. Lonn, et al., The expression of $\mathrm{NAD}(\mathrm{P}) \mathrm{H}$ :quinone oxidoreductase 1 is high in human adipose tissue, reduced by weight loss, and correlates with adiposity, 
insulin sensitivity, and markers of liver dysfunction, J Clin Endocrinol Metab 92 (2007) 2346-2352.

[15] D. Siegel, W.A. Franklin, D. Ross, Immunohistochemical detection of $\mathrm{NAD}(\mathrm{P}) \mathrm{H}$ :quinone oxidoreductase in human lung and lung tumors, Clin Cancer Res. 4 (1998) 2065-2070.

[16] A. Gaikwad, D.J. Long, 2nd, J.L. Stringer, A.K. Jaiswal, In vivo role of $\mathrm{NAD}(\mathrm{P}) \mathrm{H}$ :quinone oxidoreductase 1 (NQO1) in the regulation of intracellular redox state and accumulation of abdominal adipose tissue, J Biol Chem. 276 (2001) 22559-22564. Epub 22001 Apr 22517.

[17] M.J. Picklo, E.K. Long, E.E. Vomhof-DeKrey, Glutathionyl systems and metabolic dysfunction in obesity, Nutr Rev. 73 (2015) 858-868. doi: 810.1093/nutrit/nuv1042. Epub 2015 Oct 1022.

[18] P. Vigilanza, K. Aquilano, S. Baldelli, G. Rotilio, M.R. Ciriolo, Modulation of intracellular glutathione affects adipogenesis in 3T3-L1 cells, J Cell Physiol 226 (2011) 2016-2024.

[19] P. Calzadilla, M. Gomez-Serrano, E. Garcia-Santos, A. Schiappacasse, Y. Abalde, J.C. Calvo, B. Peral, et al., N-Acetylcysteine affects obesity-related protein expression in 3T3-L1 adipocytes, Redox Rep 18 (2013) 210-218.

[20] H.M. Findeisen, F. Gizard, Y. Zhao, H. Qing, K.L. Jones, D. Cohn, E.B. Heywood, et al., Glutathione depletion prevents diet-induced obesity and enhances insulin sensitivity, Obesity (Silver Spring) 19 (2011) 2429-2432. 
[21] E.E. Vomhof-Dekrey, M.J. Picklo, Sr., The Nrf2-antioxidant response element pathway: a target for regulating energy metabolism, J Nutr Biochem 23 (2012) 1201-1206.

[22] E.K. Long, T.C. Murphy, L.J. Leiphon, J. Watt, J.D. Morrow, G.L. Milne, J.R. Howard, et al., Trans-4-hydroxy-2-hexenal is a neurotoxic product of docosahexaenoic (22:6; n-3) acid oxidation, J Neurochem 105 (2008) 714-724.

[23] J. Ahn, H. Lee, S. Kim, T. Ha, Curcumin-induced suppression of adipogenic differentiation is accompanied by activation of Wnt/beta-catenin signaling, Am $\mathrm{J}$ Physiol Cell Physiol 298 (2010) C1510-1516.

[24] R. Dringen, B. Pfeiffer, B. Hamprecht, Synthesis of the antioxidant glutathione in neurons: supply by astrocytes of CysGly as precursor for neuronal glutathione, $\mathrm{J}$ Neurosci 19 (1999) 562-569.

[25] K. Schroder, K. Wandzioch, I. Helmcke, R.P. Brandes, Nox4 acts as a switch between differentiation and proliferation in preadipocytes, Arterioscler Thromb Vasc Biol. 29 (2009) 239-245. doi: 210.1161/ATVBAHA.1108.174219. Epub 172008 Dec 174214.

[26] A.H. Lin, H.W. Chen, C.T. Liu, C.W. Tsai, C.K. Lii, Activation of Nrf2 is required for up-regulation of the pi class of glutathione S-transferase in rat primary hepatocytes with L-methionine starvation, J Agric Food Chem 60 (2012) 65376545. 
[27] N.A. Ali, F.S. Sharopov, M. Alhaj, G.M. Hill, A. Porzel, N. Arnold, W.N. Setzer, et al., Chemical composition and biological activity of essential oil from Pulicaria undulata from Yemen, Nat Prod Commun 7 (2012) 257-260.

[28] J. Sharifi-Rad, A. Miri, S.M. Hoseini-Alfatemi, M. Sharifi-Rad, W.N. Setzer, A. Hadjiakhoondi, Chemical composition and biological activity of Pulicaria vulgaris essential oil from Iran, Nat Prod Commun 9 (2014) 1633-1636.

[29] C. Proestos, M. Komaitis, Analysis of Naturally Occurring Phenolic Compounds in Aromatic Plants by RP-HPLC Coupled to Diode Array Detector (DAD) and GCMS after Silylation, Foods 2 (2013) 90-99.

[30] E.D. Rosen, O.A. MacDougald, Adipocyte differentiation from the inside out, Nat Rev Mol Cell Biol 7 (2006) 885-896.

[31] N. Sakurai, K. Mochizuki, H. Kameji, M. Shimada, T. Goda, (-)-Epigallocatechin gallate enhances the expression of genes related to insulin sensitivity and adipocyte differentiation in 3T3-L1 adipocytes at an early stage of differentiation, Nutrition 25 (2009) 1047-1056.

[32] J.D. Hayes, A.T. Dinkova-Kostova, The Nrf2 regulatory network provides an interface between redox and intermediary metabolism, Trends Biochem Sci 39 (2014) 199-218.

[33] H.C. Ku, H.H. Chang, H.C. Liu, C.H. Hsiao, M.J. Lee, Y.J. Hu, P.F. Hung, et al., Green tea (-)-epigallocatechin gallate inhibits insulin stimulation of 3T3-L1 
preadipocyte mitogenesis via the 67-kDa laminin receptor pathway, Am J Physiol Cell Physiol 297 (2009) C121-132.

[34] H. Lee, S. Bae, Y. Yoon, The anti-adipogenic effects of (-)epigallocatechin gallate are dependent on the WNT/beta-catenin pathway, J Nutr Biochem 24 (2013) $1232-1240$.

[35] H.S. Liu, Y.H. Chen, P.F. Hung, Y.H. Kao, Inhibitory effect of green tea (-)epigallocatechin gallate on resistin gene expression in 3T3-L1 adipocytes depends on the ERK pathway, Am J Physiol Endocrinol Metab 290 (2006) E273281. 
Table 1. Primer sequences used in the study

\begin{tabular}{|lll|}
\hline $\begin{array}{l}\text { Gene } \\
\text { name }\end{array}$ & forward primer Sequence & reverse primer Sequence \\
\hline -actin & TGTCCACCTTCCAGCAGATGT & AGCTCAGTAACAGTCCGCCTAGA \\
\hline NQO1 & TATCCTTCCGAGTCATCTCTAGCA & TCTGCAGCTTCCAGCTTCTTG \\
\hline GLC $C$ & CATCGACCTGACCATCGATAAG & AGGGTGAGTGGGTCTCTAATAA \\
\hline GR & CTT TCA GCT GGA GGA CTT GC & CCA GGC CTG ATG ATG TCT TT \\
\hline GPx1 & GGG ACT ACA CCG AGA TGA ACG A & ACC ATT CAC TTC GCA CTT CTC A \\
\hline GP×3 & GCCAGCTACTGAGGTCTGACAGA & CCACCTGGTCGAACATACTTGAG \\
\hline GPx4 & TCT GGC AGG CAC CAT GTG T & CGG GCA TGC AGA TCG ACT A \\
\hline SOD1 & TGG GTT CCA CGT CCA TCA GTA & ACC GTC CTT TCC AGC AGT CA \\
\hline SOD2 & ATT AAC GCG CAG ATC ATG CA & TGT CCC CCA CCA TTG AAC TT \\
\hline CAT & GCG TCC AGT GCG CTG TAG A & TCA GGG TGG ACG TCA GTG AA \\
\hline
\end{tabular}


Table 2. Determination of bioactive fractions of Pulicaria jaubertii

\begin{tabular}{|c|c|c|c|c|c|}
\hline \multirow[b]{3}{*}{ Endpoint } & \multirow[b]{2}{*}{ Control } & \multicolumn{4}{|c|}{ Fraction $^{1}$} \\
\hline & & $\mathbf{P} \mathbf{J}_{\mathrm{M}}$ & $\mathbf{P J}_{\mathrm{H}}$ & $\mathbf{P J}_{\mathrm{D}}$ & $\mathbf{P J}_{\mathbf{A}}$ \\
\hline & & & & & \\
\hline $\begin{array}{l}\text { Triacylglyceride }{ }^{2} \\
\text { (mg/mg protein) }\end{array}$ & $1.45(0.18)$ & $0.61(0.15)^{*}$ & $1.15(0.24)$ & $0.90(0.07)^{*}$ & $1.01(0.27)$ \\
\hline $\begin{array}{l}\mathbf{G S H}^{3} \\
\text { (nmol/mg protein) }\end{array}$ & 28.5 (2.3) & $10.9(1.2)^{*}$ & $14.2(2.5)^{*}$ & $12.2(3.7)^{*}$ & $24.5(4.4)$ \\
\hline $\begin{array}{l}\text { GSSG }^{3} \\
\text { (nmol/mg protein) }\end{array}$ & $0.53(0.12)$ & $0.95(0.29)$ & $1.03(0.24)$ & $1.01(0.22)$ & $0.65(0.18)$ \\
\hline $\begin{array}{l}\text { MTT Activity } \\
\text { Gene Expression }\end{array}$ & 100 & $111(4)$ & $109(7)$ & $98(10)$ & $100(6)$ \\
\hline NQO1 & 1.00 & $6.2(0.8)^{*}$ & $2.4(0.5)$ & $7.6(0.8)^{*}$ & $1.6(0.1)$ \\
\hline GR & 1.00 & $2.7(0.1)^{*}$ & $1.7(0.1)$ & $3.3(0.5)^{*}$ & $1.3(0.1)$ \\
\hline $\mathrm{GCL}_{\mathrm{C}}$ & 1.00 & $5.7(0.1)^{*}$ & $1.6(0.5)$ & $7.1(1.2)^{*}$ & $1.4(0.4)$ \\
\hline GPx1 & 1.00 & $1.2(0.2)$ & $1.1(0.1)$ & $1.5(0.6)$ & $1.5(0.7)$ \\
\hline GPx3 & 1.00 & $7.0(0.5)^{*}$ & $5.7(0.1)^{\star}$ & $12.2(0.9)^{*}$ & $1.4(0.1)$ \\
\hline GPx4 & 1.00 & $1.6(0.2)^{*}$ & $1.3(0.2)$ & $1.7(0.2)^{*}$ & $1.6(0.0)$ \\
\hline Catalase & 1.00 & $3.2(0.3)^{*}$ & $2.8(0.7)^{*}$ & $2.9(0.3)^{*}$ & $1.6(0.0)$ \\
\hline SOD1 & 1.00 & $1.4(0.5)$ & $1.5(0.5)$ & $1.1(0.4)$ & $1.6(0.1)$ \\
\hline SOD2 & 1.00 & $1.5(0.4)$ & $1.5(0.2)$ & $1.5(0.1)$ & $1.1(0.1)$ \\
\hline
\end{tabular}

${ }^{*}$ significantly $(p \leq 0.05)$ different from the Control (vehicle only) treatment using one way ANOVA.

${ }^{1}$ Pulicaria jaubertii methanol fraction $\left(P J_{M}\right)$; PJ hexane fraction $\left(P J_{H}\right)$; PJ dichloromethane fraction $\left(P J_{D}\right)$; $P J$ aqueous fraction $\left(P J_{A}\right)$; $N A D P H ; Q u i n o n e$ Oxidoreductase 1 (NQO1); Glutathione reductase (GR); glutamate cysteine ligase catalytic unit (GCLc); Glutathione peroxidase (GPx); Superoxide dismutase (SOD). 
${ }^{2}$ Triacylglyceride content was measured after treating 3T3-L1 preadipocytes with the fractions following the seven-day differentiation regimen. Data are the mean \pm the SD for three independent experiments $(n=3)$.

${ }^{3} \mathrm{GSH}$ and GSSG were measured following a $6 \mathrm{hr}$ exposure of 3T3-L1 preadipocytes to the fractions. Data are the mean \pm the SD for three independent experiments $(n=3)$.

${ }^{4}$ MTT activity as a measure of cell viability was measured $24 \mathrm{hrs}$ after exposure to the fractions. Data are the mean \pm the SD for three independent experiments $(n=3)$ performed in quadruplicate.

${ }^{5}$ Gene expression analysis was performed on 3T3-L1 preadipocytes 48 hrs following a single exposure to the fractions. Data are the mean \pm the SD for three independent experiments $(n=3)$. 


\section{Figure Legends}

Figure 1. Effect of methanolic extract of Pulicaria Jaubertii $\left(P J_{M}\right)$ on preconfluent 3T3-L1 preadipocytes. Cell were exposed to $P J_{M}(1-250 \mu \mathrm{g} / \mathrm{ml})$ for $24 \mathrm{~h}$. Cell viability was determined using the MTT colorimetric assay. All data are reported as the percentage change in comparison with the control cells The values represent means \pm SD for three independent experiments $(n=3) .{ }^{*}=$ significantly different $(p \leq 0.05)$ from control $(0 \mu \mathrm{g} / \mathrm{mL})$ using one way ANOVA.

Figure 2. Pulicaria jaubertii extract reduces TG synthesis but not adipocyte differentiation. Preadipocytes were differentiated into adipocytes with and without treatment with the methanolic $\mathrm{PJ}$ extract $\left(\mathrm{PJ}_{\mathrm{M}}\right)$ at increasing concentrations on day 0,2 , 5, and 7. The following cells were processed for (A) Oil red O staining, (B) TG content, (C) FABP4 content, and (D) leptin content. In (A) note the decrease in Oil red $O$ with $P J_{M}$ treatment that is reflected by decreased in TG content (B). Expression of the adipocyte marker FABP4 is not blocked by $P J_{M}$ exposure (C). Leptin secretion is reduced but not abolished by PJ treatment (D). Sample sets with different letters are significantly different $(p \leq 0.05)$ using one way ANOVA. The values represent means \pm SD for three independent experiments $(n=3)$.

Figure 3. Expression of NQO1 is elevated by PJ exposure. Preadipocytes were differentiated into adipocytes with and without treatment with the methanolic PJ extract $\left(P J_{M}\right)$ at increasing concentrations on day $0,2,5$, and 7. Followed harvest, expression of the NQO1 gene $(A)$ and levels of NQO1 protein $(B)$ were determined by PJ extract in differentiated 3T3-L1. The cells were treated with control vehicle or $P J_{M} 55 \mu \mathrm{g} / \mathrm{mL}$ at day 
0,2,5,7. A representative western blot for NQO1 is provided in (B). The data represent the means $\pm S D$ of 2 different experiments performed in triplicate. Sample sets with different letters are significantly different $(p \leq 0.05)$ using one way ANOVA.

Figure 4. Pulicaria jaubertii extract exposure reduces GSH content. The effects of the $P J_{M}$ and its fractions on (A) GSH depletion (A) GSSG level (B) and GSH/GSSG ratio (C) in 3T3 L1 cells. Confluent 3T3-L1 preadipocytes were treated with increasing concentrations of $P J_{M}$ for $6 \mathrm{~h}$. The lines show significant differences between different groups $(p<0.05)$. The values represent means \pm SD for three independent experiments $(n=3) .{ }^{*}=$ significantly different $(p \leq 0.05)$ from control $(0 \mu \mathrm{g} / \mathrm{mL})$ using one way ANOVA.

Figure 5. GC-MS total ion current chromatograms of methanol extract and its fractions without TMS derivatization. (A) methanol extract $\left(P J_{M}\right),(B) D C M$ fraction $\left(P J_{D}\right),(C)$-hexane fraction $\left(P J_{H}\right),(D)$ water/methanol fraction $\left(P J_{A}\right)$. All chromatograms are normalized to the response of internal standard, thus providing proportional response based on the quantities extracted.

Figure 6 . GC-MS total ion current chromatograms of methanol extract and its fractions with TMS derivatization. $(A)$ methanol extract $\left(P J_{M}\right),(B) D C M$ fraction $\left(P J_{D}\right)$, (C) n-hexane fraction $\left(P J_{H}\right)$, (D) water/methanol fraction $\left(P J_{A}\right)$. Note that analysis of TMS derivatives allowed for enhanced characterization of the fractions and allowed for identification of catechin-like compounds in the methanol extract and the DCM fraction. All chromatograms are normalized to the response of internal standard, thus providing proportional response based on quantities extracted. 
Figure 7. GC-MS characterization of DCM fraction ( $\left.P J_{D}\right)$ and EI-MS spectrum of most abundant catechin-like compounds. (A) Total ion current chromatogram (B-G) EI-MS spectra of peaks D1-D6. Compounds D2, D3, D4 showed high identity (> 80\%) with the EI/MS NIST library for catechin. Compounds were analyzed as TMS derivatives.

Figure 8. GC-MS characterization of catechin and epicatechin standards. (A) TMSderivatives of catechin and epicatechin standards were analyzed by GC-MS in comparison to the TMS-derivatized DCM fraction, $\mathrm{PJ}_{\mathrm{D}}$. Note that the retention times for the standards do not match that of the compounds observed in the $P J_{D}$. The EI-MS spectra of catechin and epicatechin are provided in $(B)$ and $(C)$. Note that of the 368.2 ions present in the standards is also enriched in the TMS-derivatized compounds observed in the DCM fraction detailed in Figure 7.

Figure 9. Extracted ion chromatograms and mass spectra of LC-HR-MS analysis of the DCM fraction $\mathbf{P J}_{\mathrm{D}}$. (A) Extracted ion chromatogram (EIC) of ions 289.07, 317.05 and 359.19. (B) Time of flight (TOF) mass spectrum of L1 peak, C) TOF mass spectra of L2 peak and mass accuracy confirming the catechin-like structure. The structure of catechin ( $\mathrm{m} / \mathrm{z} 289.0718)$ is provided for comparison. 


\section{Figure 1}

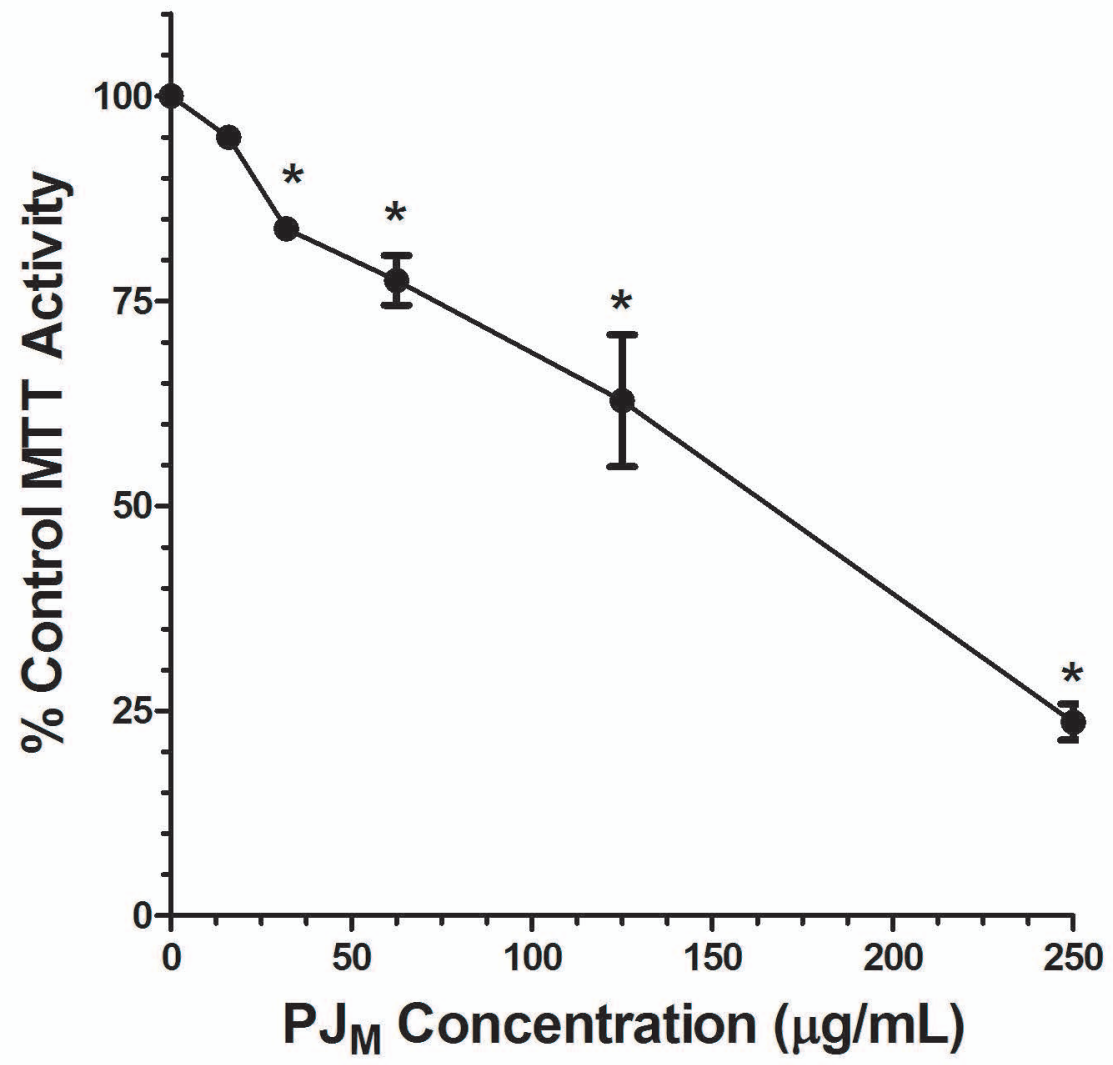


Figure 2
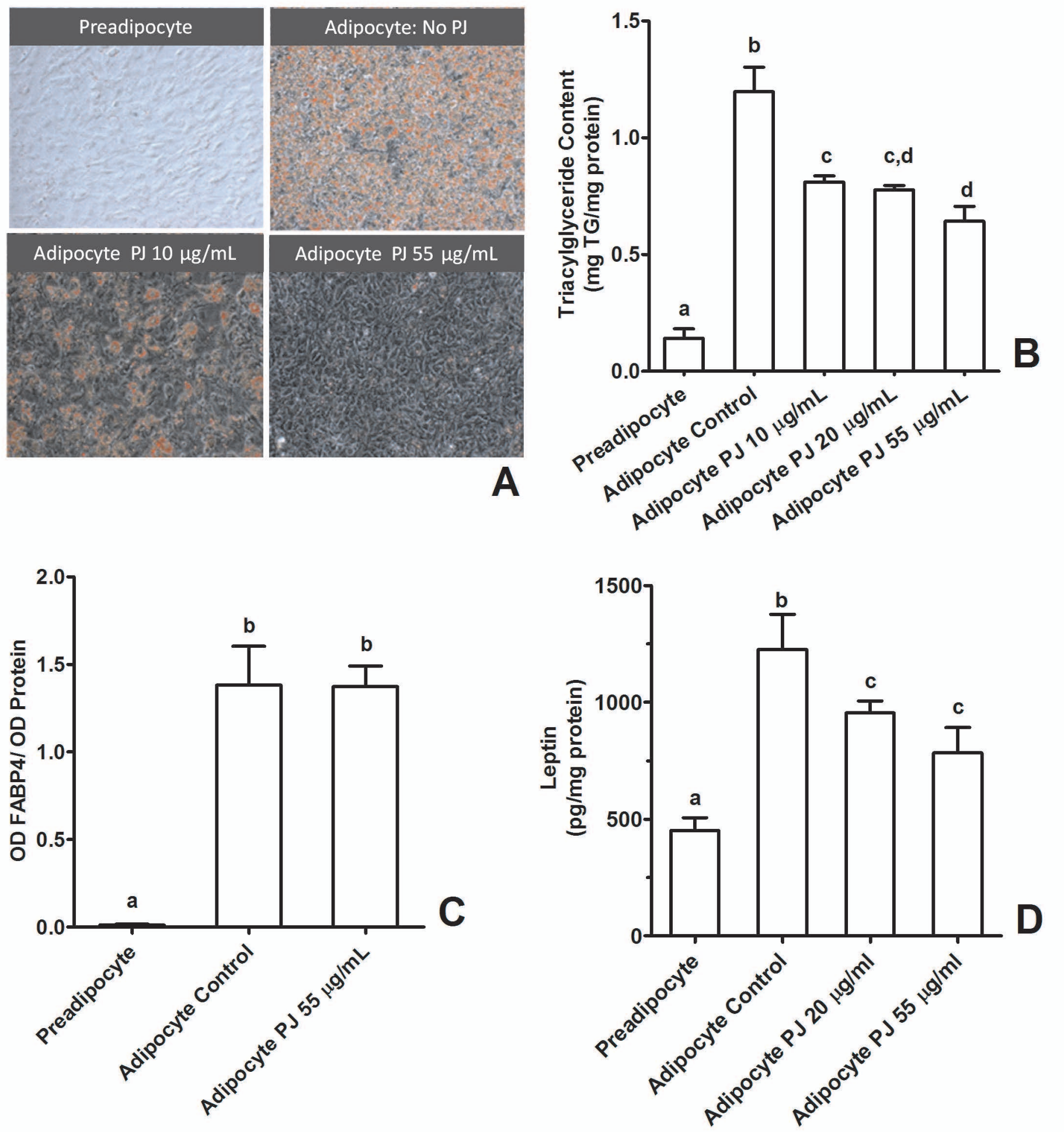
Figure 3

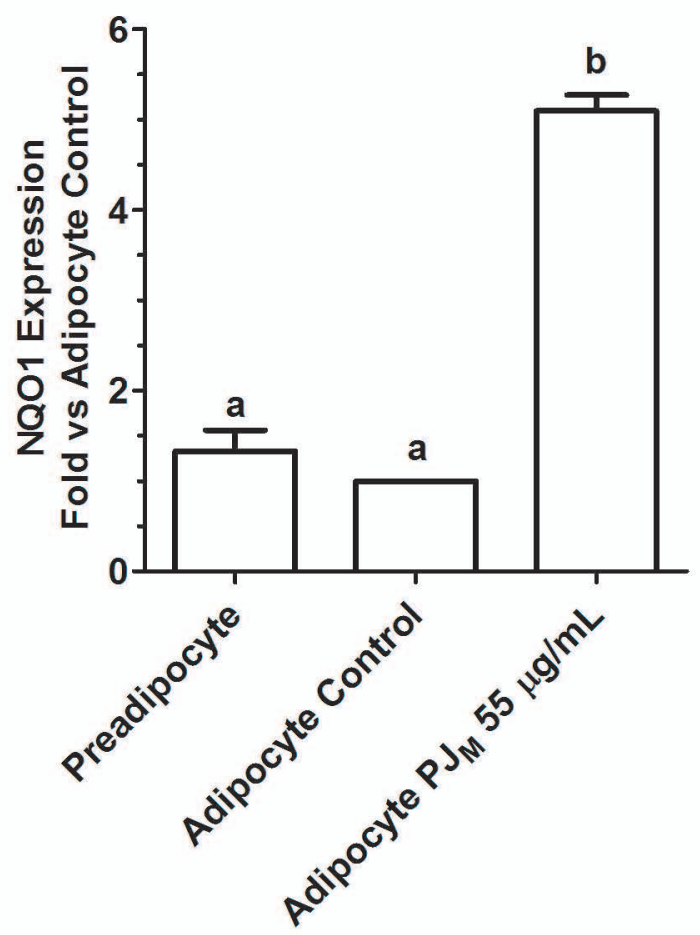

A

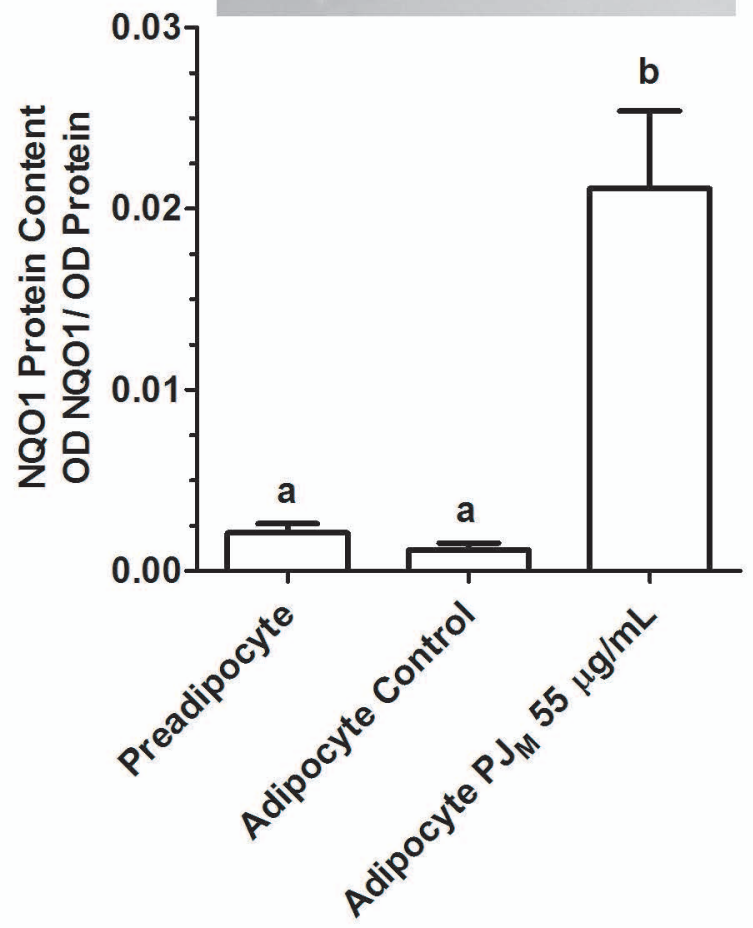

B 
Figure 4
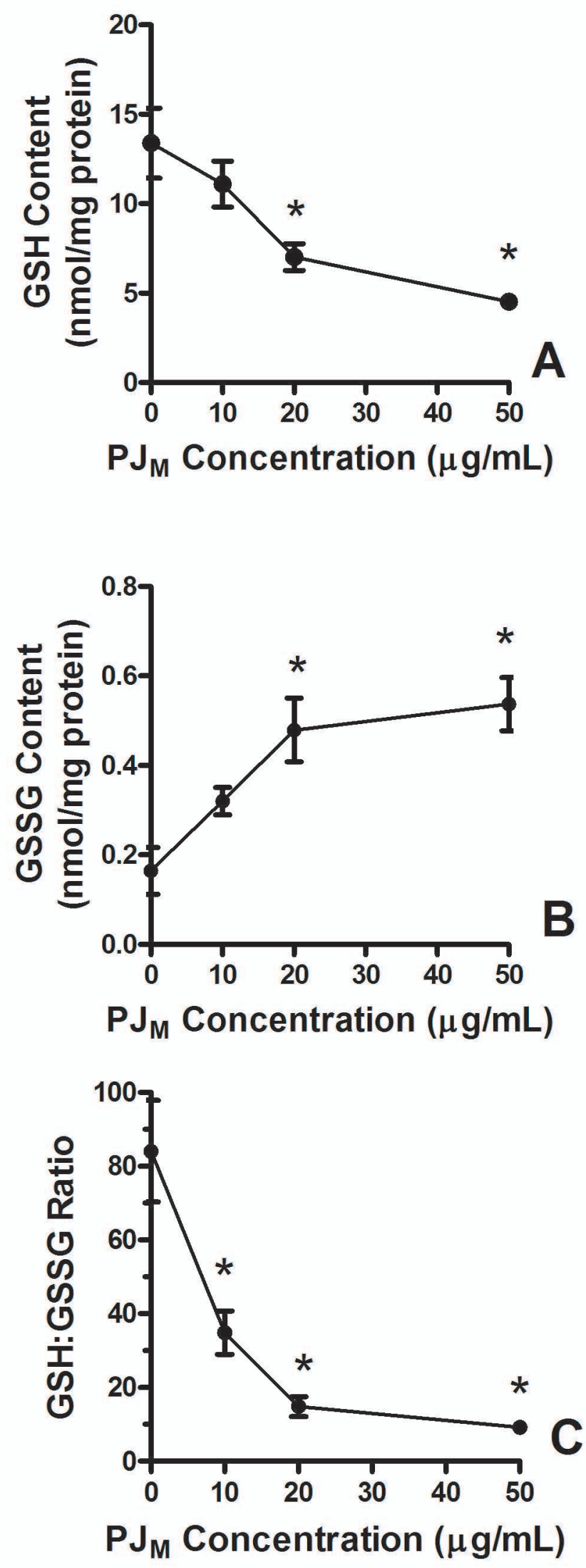
Figure 5.

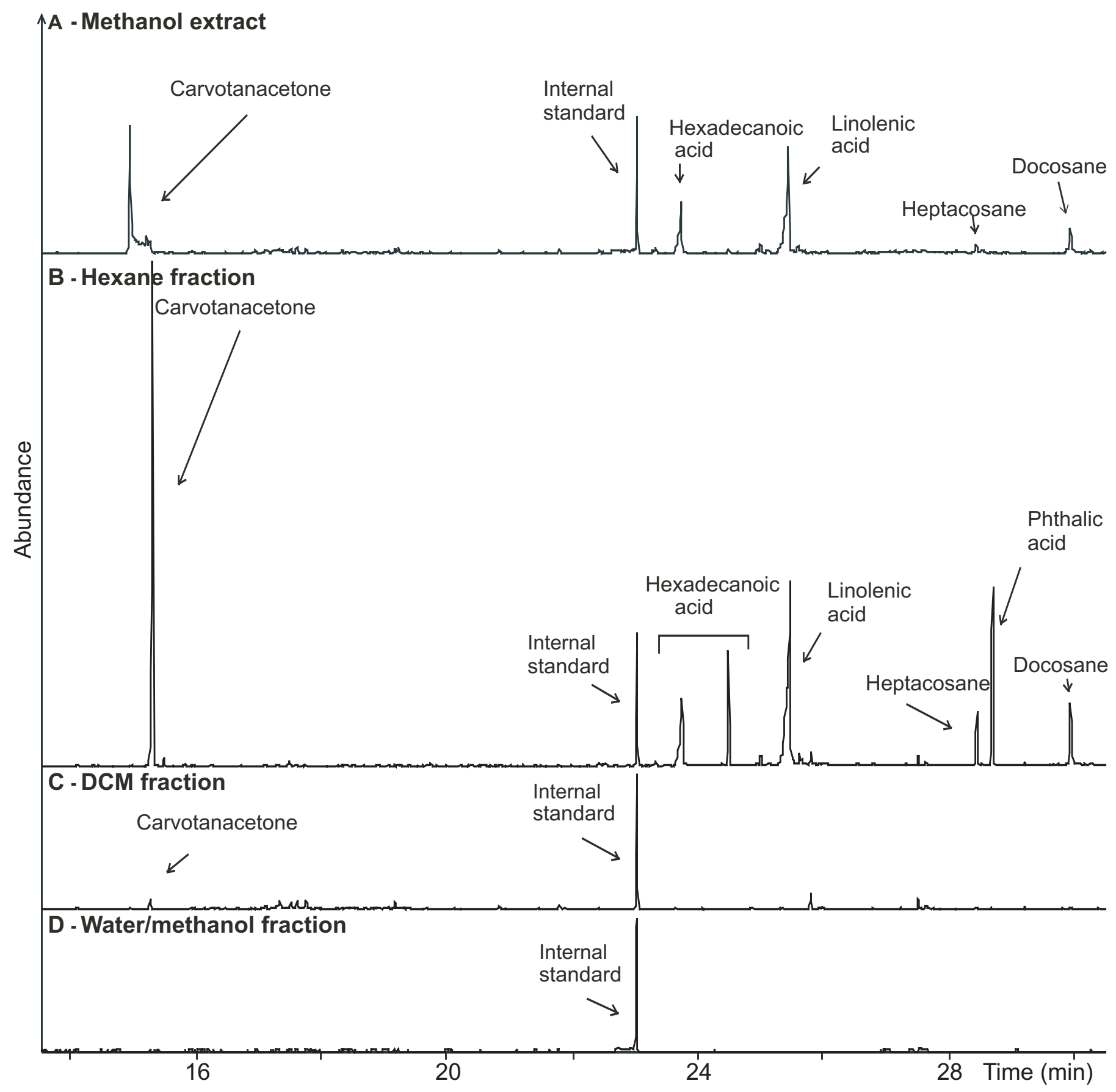




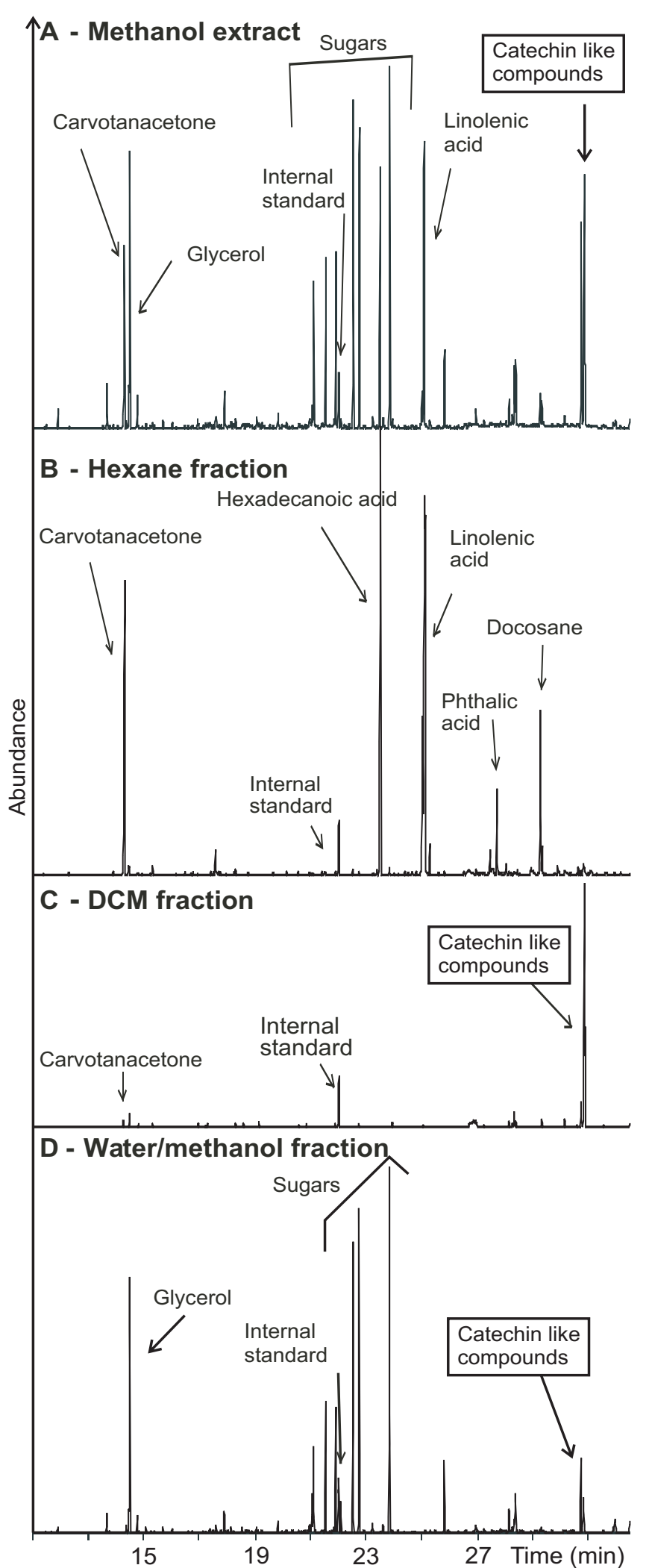

Figure 6 
Figure 7
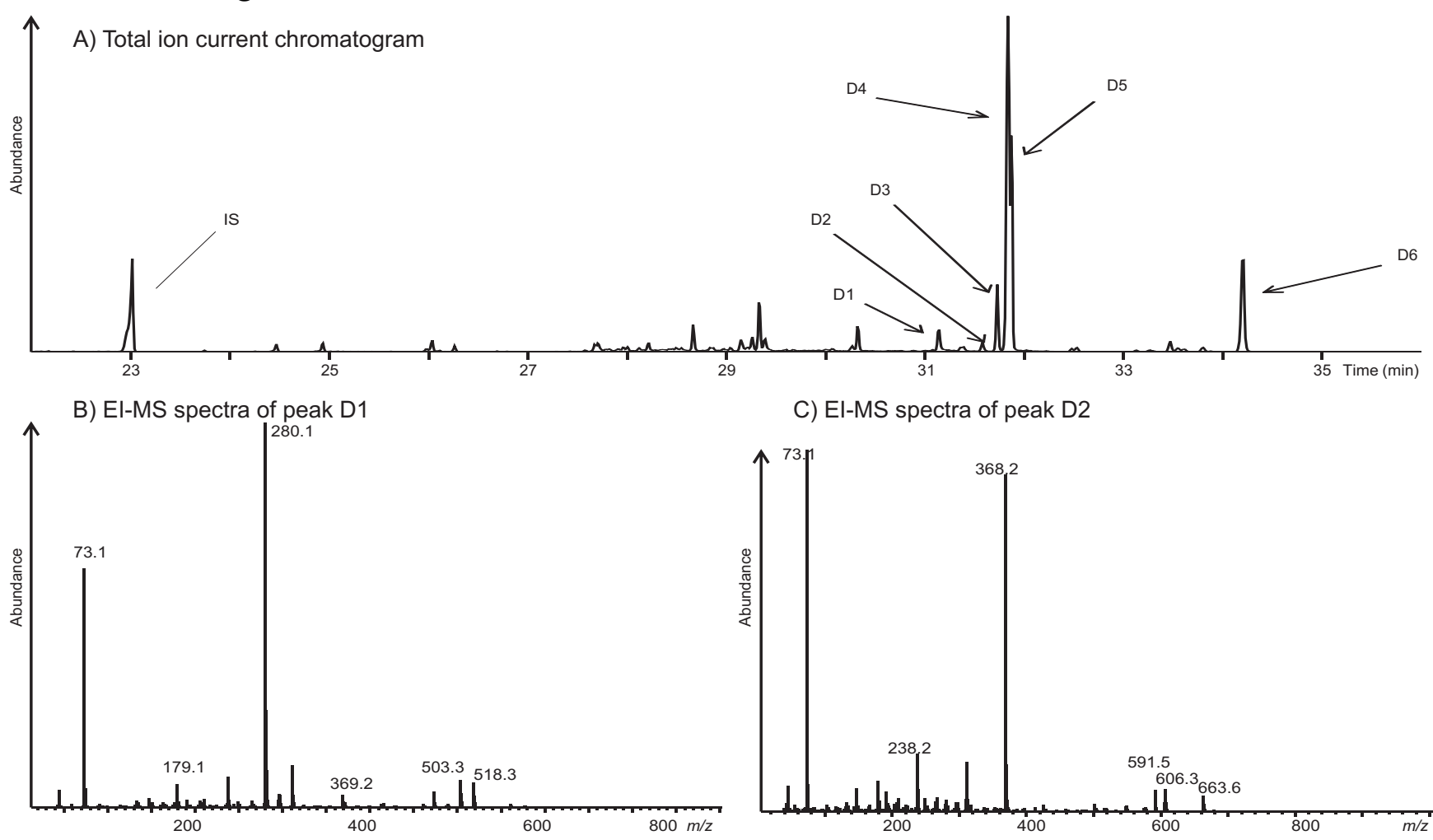

C) El-MS spectra of peak D2

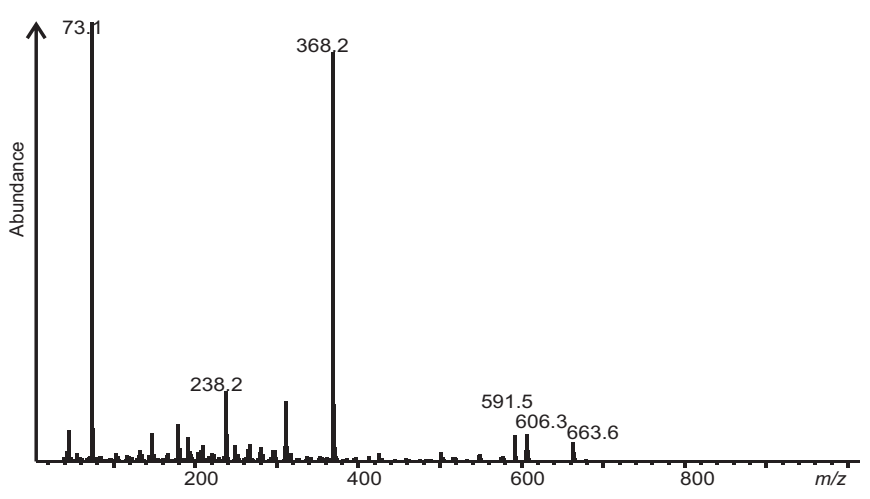

D) El-MS spectra of peak D3
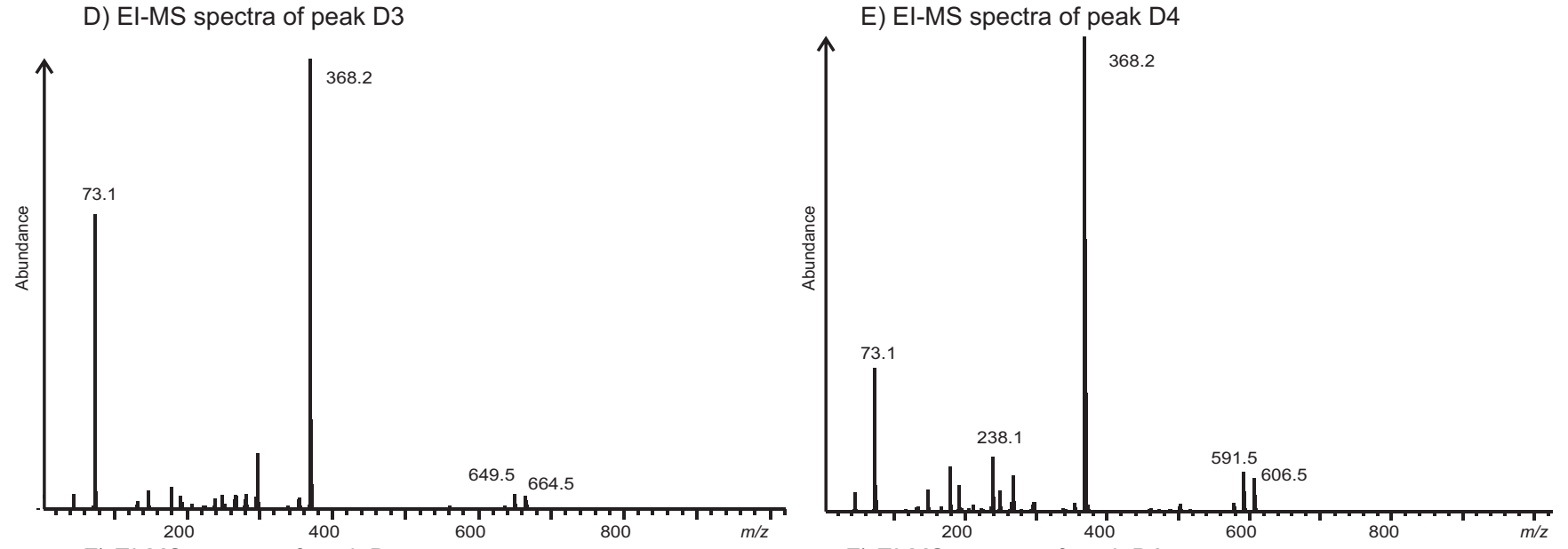

F) El-MS spectra of peak D5

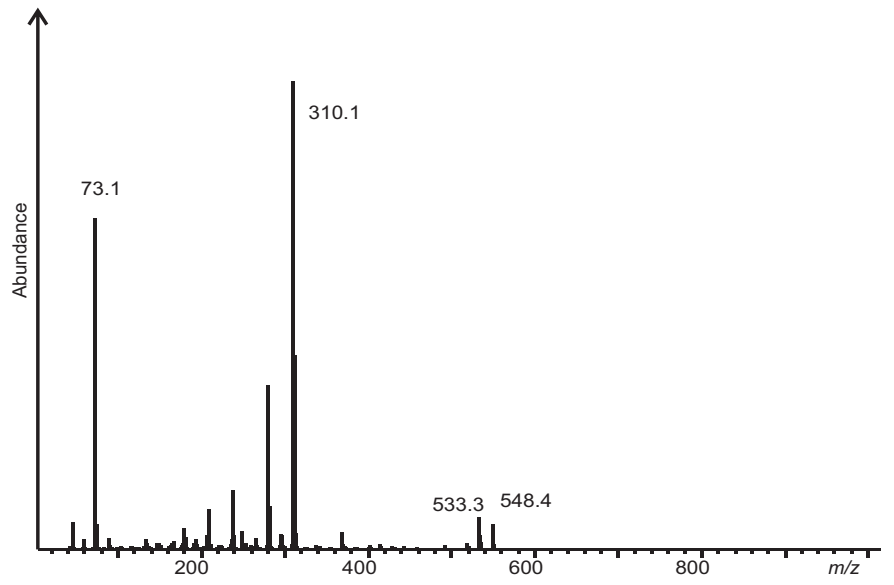

F) El-MS spectra of peak D6

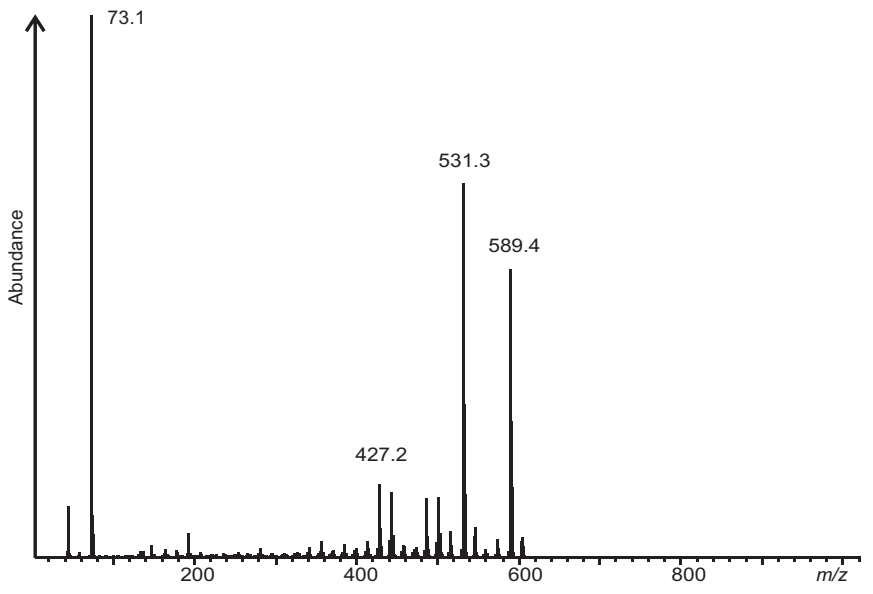


Figure 8.

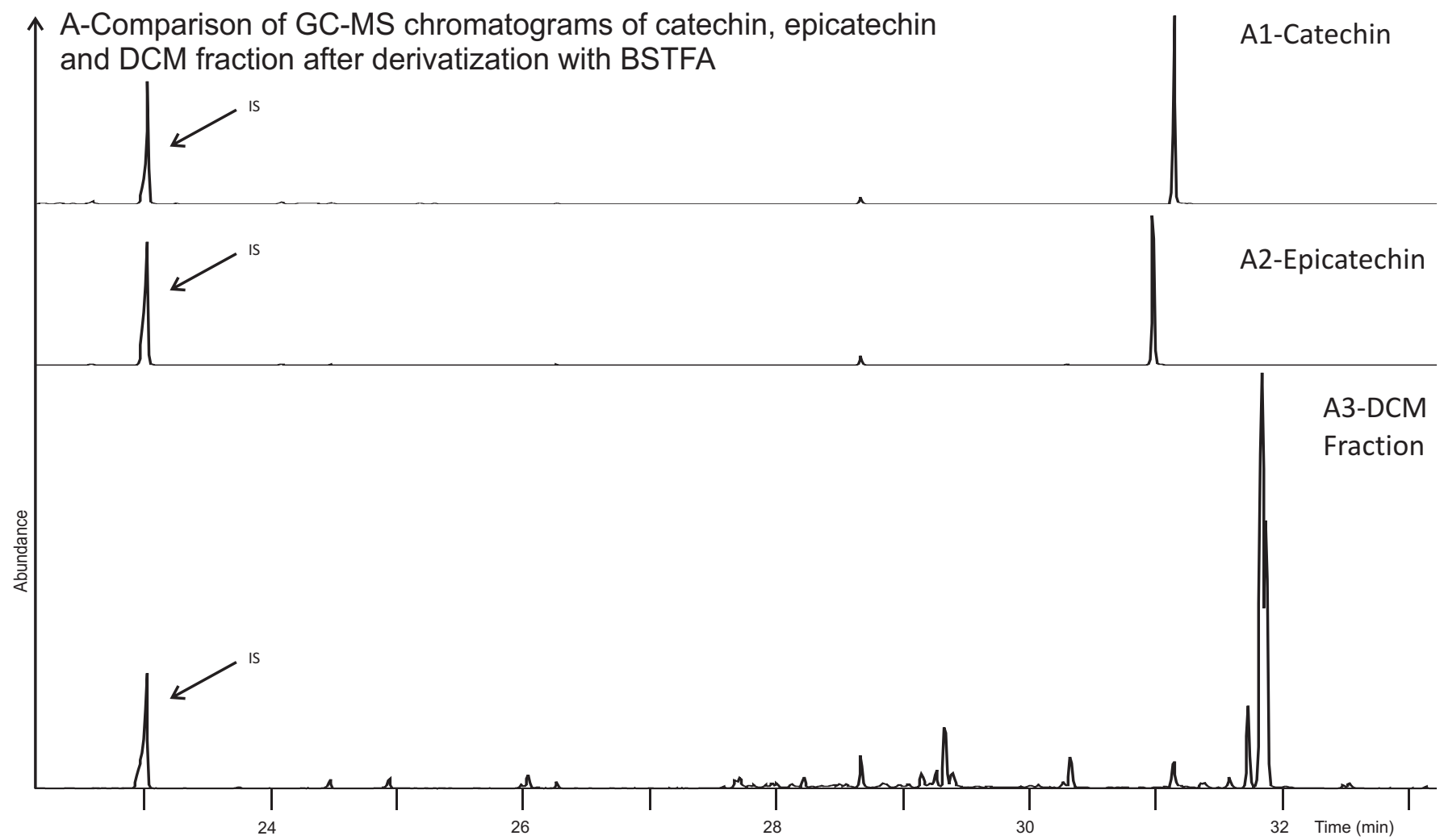

B) EI-MS spectra of catechin standard

C) El-MS spectra of epicatechin standard
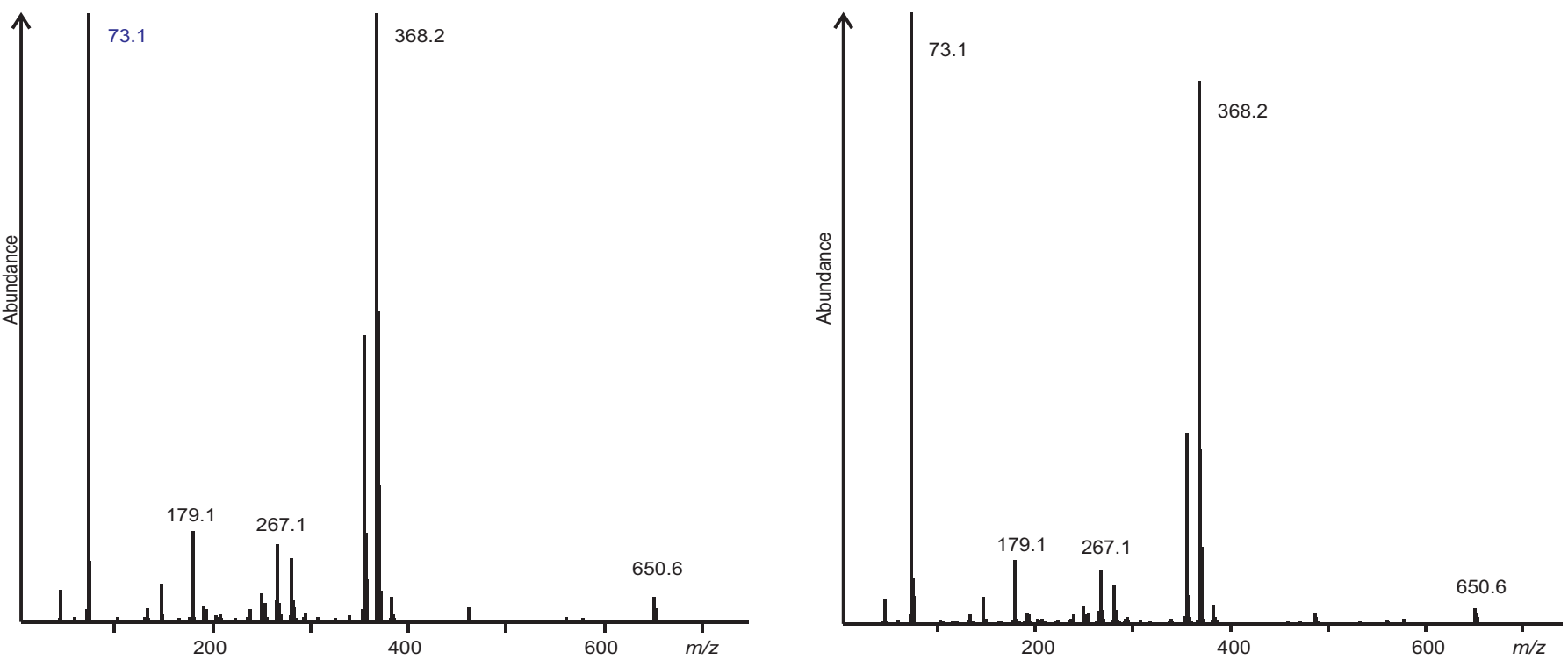
Figure 9

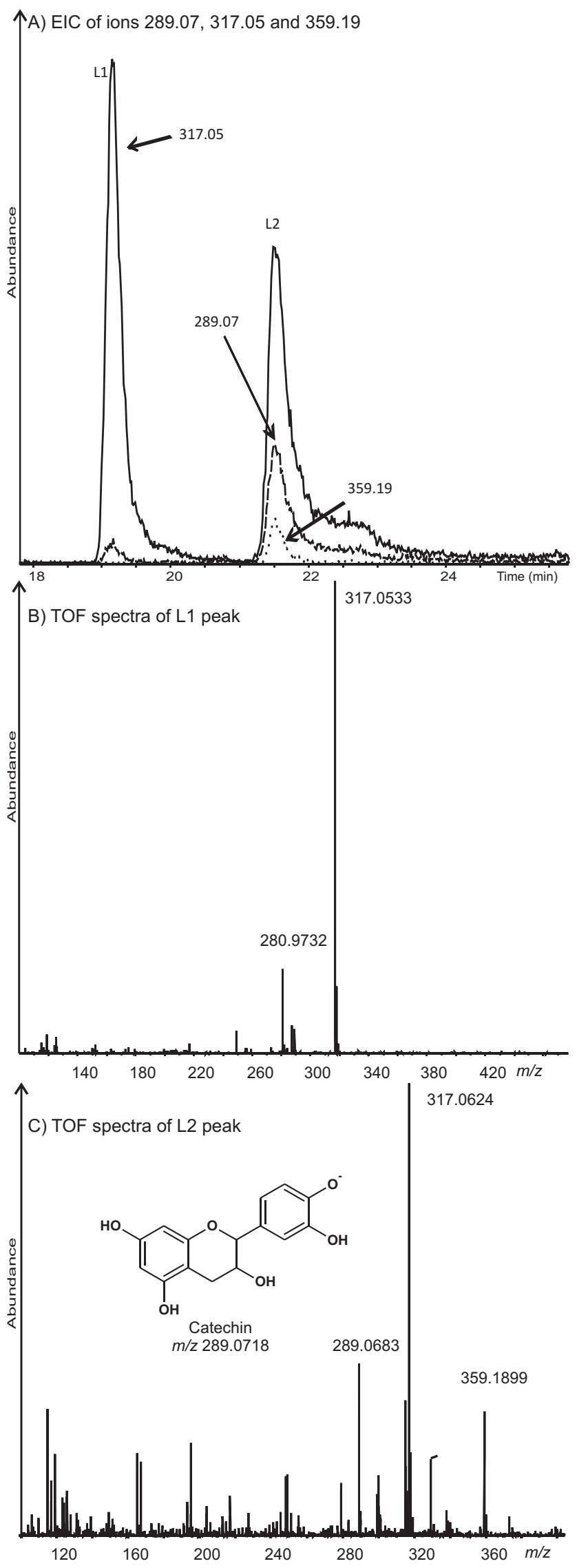

